\title{
Evaluation of the Safety and Efficacy of Selective Retina Therapy Laser Treatment in Patients with Central Serous Chorioretinopathy
}

\author{
Jaeryung $\mathrm{Oh}^{1 *}$, Chang Ki Yoon ${ }^{2 *}$, Bo Hee Kim², Hyeong Gon $\mathrm{Yu}^{2}$ \\ ${ }^{1}$ Department of Ophthalmology, Korea University Anam Hospital, Seoul, Korea \\ ${ }^{2}$ Department of Ophthalmology, Seoul National University Hospital, Seoul, Korea
}

\begin{abstract}
Purpose: To assess the safety and efficacy of selective retina therapy (SRT) using a Q-switched neodymium-doped yttrium lithium fluoride laser with feedback systems in patients with idiopathic central serous chorioretinopathy (CSC).

Methods: This randomized clinical trial enrolled patients having at least 3-month symptom of CSC. From month 3 visit, all subjects in both groups were eligible for SRT retreatment if they showed persistent or recurrent subretinal fluid (SRF). The primary outcome was complete resolution of SRF by optical coherence tomography at 3 months after treatment. The secondary outcomes were changes in SRF, central macular thickness (CMT) and best-corrected visual acuity at the 1-, 3-, and 6-month examinations.

Results: Sixty-eight CSC patients were included (SRT, 31; control, 37). After 1 and 3 months, complete resolution of SRF was achieved in $25.8 \%$ and $54.8 \%$ of SRT group and $17.6 \%$ and $35.1 \%$ of controls. The differences were not statistically significant ( $p=0.424$ and $p=0.142$, respectively). However, mixed model for repeated measures analyses showed that the reduction of SRF and CMT were observed earlier in SRT group than in the sham group (least squares mean difference, $-59.7 \mu \mathrm{m} ; 95 \%$ confidence interval, -98.2 to $-21.2 ; p=0.0029$; least squares mean difference $-67.0 \mu \mathrm{m} ; 95 \%$ confidence interval, -104.8 to -29.2 ; $p=0.0007$, respectively). Significant reduction of SRF ( $\geq 50 \%$ reduction from baseline) was more frequently observed in SRT group (80.6\%) than the sham group (44.1\%) at month $1(p=0.007)$. Early reduction of SRF and CMT was more abundant in SRT group with symptom duration less than 6 months. Treatment related serious adverse events were not observed.

Conclusions: SRT using a Q-switched neodymium-doped yttrium lithium fluoride laser with feedback system was safe in this trial and effective for early resolution of SRF in the CSC patients. Early intervention with SRT can be a safe alternative for patients with acute symptomatic CSC.
\end{abstract}

Key Words: Central serous chorioretinopathy, Clinical trial, Laser treatment, Subthreshold micropulse laser

Received: August 26, 2020 Final revision: November 5, 2020 Accepted: November 30, 2020

Corresponding Author: Hyeong Gon Yu, MD. Department of Ophthalmology, Seoul National University Hospital, 101 Daehak-ro, Jongno-gu, Seoul 03080, Korea. Tel: 82-2-2072-2437, Fax: 82-2-741-3187, E-mail: hgonyu@snu.ac.kr

*These two authors contributed equally to this work. 
Central serous chorioretinopathy (CSC) is chorioretinal disorder characterized by neurosensory retinal detachment of the central retina. CSC is three-fold more prevalent in middle aged males than in females [1]. Multifactorial etiologies have been suggested, including hyperpermeability of the choroid or dysfunction of retinal pigment epithelium (RPE). Majority of cases regress spontaneously within 3 to 4 months with good visual recovery [2]. However, about half of patients develop recurrence within one year from the first episode [3,4]. Irreversible complications including RPE atrophy, subretinal fibrosis and choroidal neovascularization (CNV) can develop and result in severe vision loss. Recurrent episodes can also impair visual function through metamorphopsia, and loss of color sensation as well as decreases in vision. Moreover, eyes having vision of $20 / 20$ or better showed reduced cone density with preserved ellipsoid zone [5]. This may explain decreased visual function other than visual acuity in CSC patients. Therefore, active treatment is required both for chronic cases and can be considered in acute cases for early recovery with less damage.

Observation for self-limiting has been mainstay of treatment for acute CSC. If CSC continues more than 3 months or faster resolution is needed, direct focal laser treatment, photodynamic therapy (PDT); mineralocorticoid receptor antagonists; and intravitreal anti-vascular endothelial growth factor (VEGF) injections are used as treatment options. Because of possible risk of adverse events and questionable efficacy of treatment, robust treatment guideline is not established yet. Direct focal laser treatment has a been used for many years, and is applied at focal leakage points. Although this hastens subretinal fluid (SRF) resolution, final visual gain is not generally improved [6]. Above all, scotoma at the irradiated area or risk of CNV formation make this treatment less favored. Subthreshold micropulse laser and selective retina therapy (SRT) are recently introduced laser approaches to treat retinal disease while minimizing collateral tissue damage.

SRT adopts a Q-switched neodymium-doped yttrium lithium fluoride (Nd: YLF) laser and real time feedback dosimetry system to selectively modulate RPE cells via microvaporization. Selective damage of RPE is suggested to help rejuvenate RPE function and improve Bruch membrane function via migration and proliferation of RPE. Recovered RPE is presumed to improve pump function and accelerate absorption of SRF in CSC [7,8]. Several studies showed favorable outcome on CSC resolution using SRT $[9,10]$. However, no prospectively designed randomized clinical trial to better characterize safety and efficacy of SRT for CSC patients has not been performed yet. Herein, the purpose of this study aims to evaluate the safety and efficacy of using SRT in CSC patients.

\section{Materials and Methods}

This study was a multicenter, randomized, sham-controlled clinical trial at seven sites in Korea. The study is registered at Clinical trials.gov (NCT03758963). This study was approved by the institutional review board at all sites. The study was conducted in accordance with the tenets of the Declaration of Helsinki. All participants provided written informed consent.

Inclusion criteria were CSC with clinical symptoms over 3 months, best-corrected visual acuity (BCVA) of $20 / 200$ or better based on Early Treatment Diabetic Retinopathy Study (ETDRS) chart, SRF involving the fovea, and $\geq 1$ to $\leq 3$ active leakage sites in fundus fluorescein angiography (FA). Patients aged 19 years or over and less than 55 years were included. Patients were excluded if they had other retinal diseases such as choroidal neovascularization or polypoidal choroidal vasculopathy, the conditions that made laser therapy difficult such as cataract or vitreous opacity, atrophy (diameter $\geq 1,000 \mu \mathrm{m}$ ) in the RPE including the fovea, previous history of laser or PDT, history of steroid treatment (periocular, sub-tenon and intraocular) within the last one year, history of intraocular injection of anti-VEGF agent, history of intraocular surgery within the last six months, or pigment epithelial detachment that was directly related to the point of leakage with a diameter of more than 1,000 micron.

Each patient underwent a complete ophthalmologic examination, including determination of BCVA using standard ETDRS charts, contrast sensitivity, color fundus photography, fundus autofluorescence (FAF), FA, indocyanine green angiography (ICGA) and optical coherence tomography (OCT). For OCT images, two different spectral domain OCTs were used; 3D-OCT 1000 (Topcon 3D-OCT; Topcon, Tokyo, Japan) and Spectralis (Heidelberg Spectralis OCT; Heidelberg Engineering, Heidelberg, Germany). We used $512 \times 128$ cube scan covering $6 \times 6 \mathrm{~mm}$ for 3D-OCT 1000 and fast macular volume scan covering $20^{\circ}$ 
$\times 20^{\circ}$ for Spectralis. The results were analyzed using a macular thickness map provided by each OCT software. During the follow-up visits, each patient was evaluated using the same OCT device used for baseline examination. FAF, FA and ICGA were obtained using a confocal scanning laser ophthalmoscope (Heidelberg Spectralis HRA+OCT, Heidelberg Engineering).

Participants were randomized $1: 1$ into the SRT group or sham group, and the randomization code was produced by block randomization. Double-masking was applied to subjects and independent efficacy evaluators. Masked independent evaluators conducted the efficacy evaluation tests including OCT, BCVA, contrast sensitivity tests, and FA. The maksing on the test devices was maintained until the database was unlocked.

Subjects underwent BCVA testing, OCT imaging, contrast sensitivity testing and slit lamp biomicroscopy prior to assigned treatment on day 0 , and underwent FA and evaluation of adverse events after treatment. Following initial treatment, all subjects had five follow-up visits at month 1, 3, 4, 5, and 6. At each of these follow-up visits, subjects underwent a complete ophthalmic examination, BCVA and contrast sensitivity assessments, slit lamp biomicroscopy, color fundus photography, OCT, FAF and FA. ICGA was performed at Screening, month 3 and 6 only.

The primary outcome was complete resolution of SRF by OCT at month 3. Complete resolution of SRF was defined when the volume of SRF on OCT was estimated as zero. Secondary efficacy endpoints included improvement of BCVA, degree of retinal functional damage, change in SRF and central macular thickness (CMT) at month 1, 3, and 6. Possible retinal functional damage was assessed using contrast sensitivity. Removal rate of leakage and recurrence rate at month 6 were also included. In addition, subgroup analysis was also performed according to the difference of symptom duration. Safety endpoints included an evaluation of all treatment emergent adverse events that occurred during the study period.

\section{SRT}

SRT was performed using the R:GEN laser system (Lutronic Corporation, Goyang, Korea), which is a Q-switched $\mathrm{Nd}$ : YLF laser $(527 \mathrm{~nm})$, pulse duration $1.7 \mu \mathrm{s}$, repetition rate of $100 \mathrm{~Hz}$, and pulse energy of 30 to $350 \mu \mathrm{J}$. Single-pulse energy was the only adjustable parameter, and the value was determined through test exposure assessment. Test exposure to determine laser energy was determined by irradiation in the upper or lower vessel arcade outside the treatment area. Since the laser spot is not visible, single-pulse energy is determined by multiple test exposures at various pulse energy levels at the test exposure site until the proper energy display is determined using the optoacoustic and reflectometry real time feedback (RTF) system that are part of the laser system. Multiple irradiations were performed around leakage (Fig. 1A, 1B). Each spot was irradiated 0.5 to 1 spot diameter apart. Foveal center of $200 \mu \mathrm{m}$ diameter was avoided. For the control group undergoing the sham procedure, the exact laser procedure was performed, except that short bursts of light from the retinal illumination system were used instead of the laser beam. Treatments were performed on the day of randomization, and each participant visited monthly. Primary endpoint was at month 3 . Subjects in the sham group were eligible to receive SRT for the first time at month 3 visit as long as these subjects showed persistent SRF that had not resolved spontaneously. From month 3 visit, all subjects in both groups were eligible for SRT retreatments at any evaluation visit if they showed persistent SRF or recurrence of SRF.

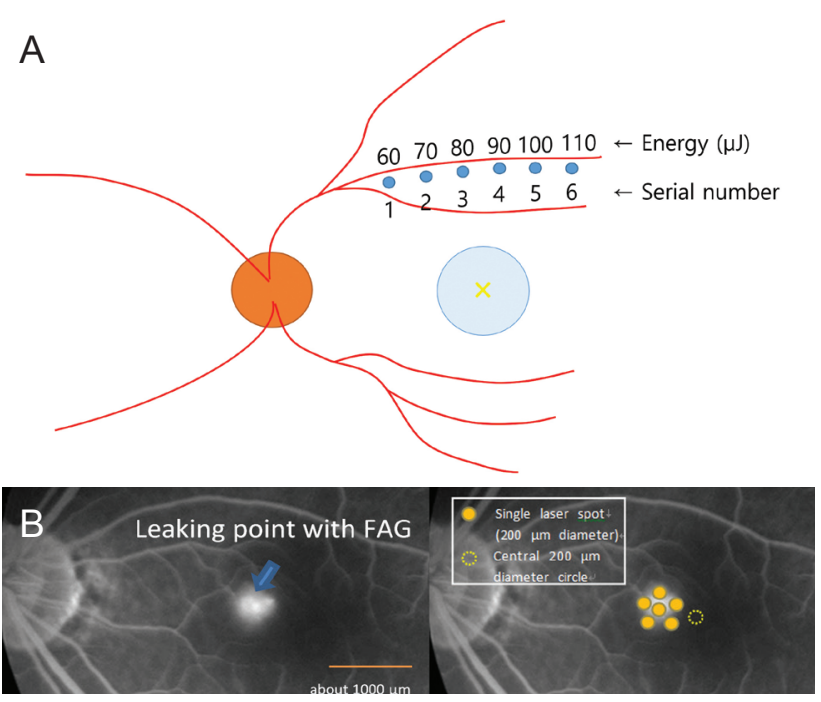

Fig. 1. Illustration of selective retina therapy test exposure to determine laser energy was determined by irradiation in the upper or lower vessel arcade outside the treatment area until the proper energy display is titrated using the optoacoustic and reflectometry feedback systems. (A) Multiple irradiations were performed around a leakage. (B) Blue arrow indicates leakage determined using fluorescein angiography (FAG) and the yellow dots indicate laser irradiated spots. 


\section{OCT measurements}

OCT imaging was evaluated by an independent OCT reading center located at Korea University Hospital. In this trial, the peak height and estimated volume of SRF, CMT, and total macular volume (TMV) were measured with an objective and standardized evaluation method for each model of OCT in use in the study, instead of unifying the OCT data across models. The peak height of SRF was determined as the highest height of SRF in OCT line scans. The volume of SRF was estimated by multiplying the peak height, number of B-scans demonstrating SRF, and distance between adjacent two B-scans. Resolution of SRF was graded based on distribution of decreased percent of estimated volume of SRF. CMT was defined as a mean retinal thickness within ETDRS central $1 \mathrm{~mm}$ area. Two experienced researchers at the OCT Reading Center analyzed the OCT images based on the method set for each OCT model.

\section{Statistical analyses}

Statistical analyses, including descriptive statistics, calculation of inferential statistics, and graphical representations were performed using IBM SPSS Statistics ver. 20.0 (IBM Corp., Armonk, NY, USA). Analysis of mixed model for repeated measures was performed using SAS ver. 9.2 (SAS Institute, Cary, NC, USA). Unless specified otherwise, all statistical tests were two-sided and a difference resulting in a $p$-value $\leq 0.05$ was considered statistically significant. Continuous variable means were compared using $t$-test or the Mann-Whitney test. And categorical variables were compared using chi-square test. Fisher exact test for multiple rows more than 2 was calculated by vassarstats (http://vassarstats.net/fisher2x3.html).

\section{Results}

\section{Demographic data and baseline characteristics}

Of the 82 participants screened for eligibility, 70 were eligible to be enrolled, provided written informed consent, and were randomized to receive SRT $(\mathrm{n}=33)$ or sham $(\mathrm{n}=$ 37) treatment. However, two subjects in the SRT group were excluded from the primary analysis. One was excluded due to an age violation and the other withdrew participation due to resolution of symptoms before treatment. Finally, data from 68 participants were analyzed for this study. The mean age of the 68 participants at baseline was 45.6 years (standard deviation, \pm 7.0 ), and they were predominantly male (79.4\%). The mean duration of symptoms was 17. 2 months (standard deviation, \pm 24.5 ). Baseline characteristics of the participants were comparable between both groups (Table 1). All 68 patients completed 3 months of follow-up, and the following additional treatments were performed subsequently. Between 3 and 5 months, 10 subjects in the SRT group received an additional treatment, and 3 subjects received two additional treatments. Beginning at 3 months, 23 subjects in the control group received SRT; 18 received a single treatment and 5 subjects received treatment twice.

\section{Resolution of SRF}

At baseline, the median height and volume of SRF of 68 patients were $167.3 \mu \mathrm{m}$ (interquartile range, 100.8 to 253.4 $\mu \mathrm{m}$ ) and $0.469 \mathrm{~mm}^{3}$ (interquartile range, 0.183 to 1.167 $\mathrm{mm}^{3}$ ). SRF decreased in $84.6 \%$ and $85.3 \%$ of patients at month 1 and month 3, respectively. However, complete resolution of SRF was noted in only $21.5 \%$ and $44.1 \%$ of patients at month 1 and 3, respectively. At month 1, 25.8\% of the SRT group and $17.6 \%$ of the sham group had achieved

Table 1. Comparison of baseline characteristics between SRT and the sham groups

\begin{tabular}{lccc}
\hline Characteristics & SRT group $(\mathrm{n}=31)$ & Sham group $(\mathrm{n}=37)$ & $p$-value \\
\hline Age (yr) & $44.9 \pm 6.9$ & $46 . \pm 7.1$ & $0.464^{*}$ \\
Sex, female & $5(16.1)$ & $9(24.3)$ & $0.405^{\dagger}$ \\
Duration symptoms (mon) & $14.8 \pm 23.7$ & $19.2 \pm 25.4$ & $0.457^{*}$ \\
\hline
\end{tabular}

Values are presented as mean \pm standard deviation or number (\%).

$\mathrm{SRT}=$ selective retina therapy.

${ }^{*}$ Independent $t$-test; ${ }^{\dagger}$ Chi-square test. 
complete resolution of SRF (Table 2). At month 3, complete resolution of SRF was noted in $54.8 \%$ in SRT group and $35.1 \%$ in the sham group. Complete resolution rate was higher in SRT group. However, the difference was not statistically significant both at month 1 and 3 ( $p=0.424, p=$ 0.142 , respectively). The SRF volume was reduced to 50.0 $\pm 64.2 \%$ (mean \pm standard deviation) at month 1 and 56.8 $\pm 111.0 \%$ at month 3. Degree of resolution of SRF was graded into 2 classes; significant $(\geq 50 \%$ SRF reduction from baseline) and moderate $(<50 \%$ SRF reduction from baseline). At month 1, degree of resolution of SRF was significantly different between SRT and sham groups $(p=$ 0.007 , Fisher exact test) (Table 2). Significant resolution of SRF ( $\geq 50 \%$ SRF reduction from baseline) was more fre-

Table 2. Comparison of resolution of subretinal fluid between the SRT group and the sham group

\begin{tabular}{lccc}
\hline & SRT group $(\mathrm{n}=31)$ & Sham group $(\mathrm{n}=37)^{p}$-value $^{*}$ \\
\hline At month 1 & & & \\
$\quad$ Complete resolution of SRF (yes : no) & $8: 23(25.8: 74.2)$ & $6: 28(17.6: 82.4)$ & 0.424 \\
$\quad$ Degree of resolution in SRF (significant ${ }^{\dagger}:$ moderate $\left.^{\ddagger}\right)$ & $25: 6(80.6: 19.4)$ & $15: 19(44.1: 55.9)$ & $0.002^{\S}$ \\
At month 3 & & & \\
$\quad$ Complete resolution of SRF (yes : no) & $17: 14(54.8: 45.2)$ & $13: 24(35.1: 64.9)$ & 0.142 \\
Degree of resolution in SRF (significant ${ }^{\dagger}:$ moderate $\left.^{\ddagger}\right)$ & $23: 8(74.2: 25.8)$ & $21: 16(56.8: 43.2)$ & 0.134 \\
\hline
\end{tabular}

Values are presented as number (\%).

$\mathrm{SRT}=$ selective retina therapy; $\mathrm{SRF}=$ subretinal fluid.

*Fisher exact test; ${ }^{\dagger}$ SRF reduction $\geq 50 \%$ from baseline; ${ }^{*}$ SRF reduction less than $50 \%$ or increased; ${ }^{\S} p<0.05$.
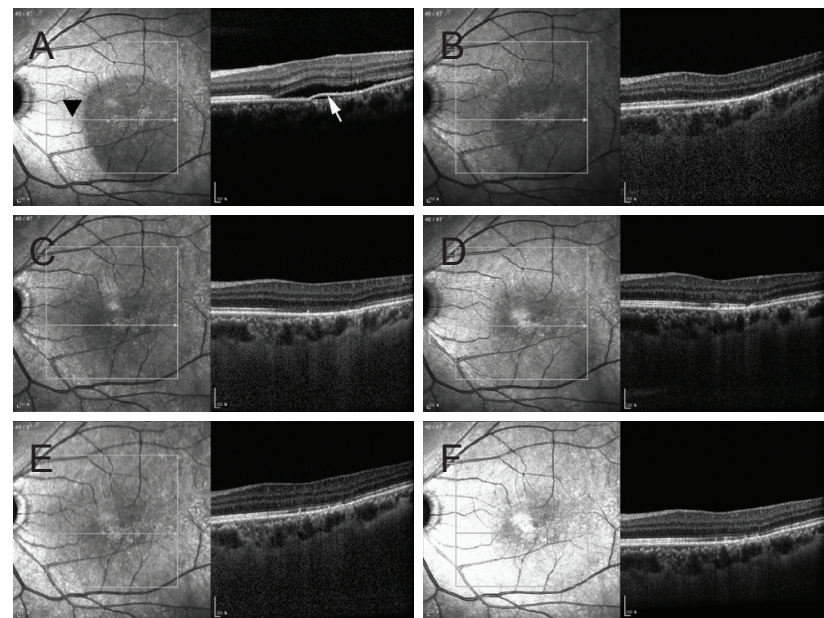

Fig. 2. Representative case of selective retina therapy (SRT) arm (subject 04-19). (A) A 54-year-old man with a 49-week history of subretinal fluid (SRF) in the left eye. Best-corrected visual acuity of the left eye was $20 / 25$ at baseline. Black arrowhead indicates the line where OCT scan was performed. Left picture is infrared reflectance image and white line indicated by black arrowhead means location where optical coherence tomography (OCT) scan was performed. SRF with flat pigment epithelial detachment (white arrow) was observed at baseline by OCT. The height of SRF was $137.5 \mu \mathrm{m}$ at the baseline. (B) SRF was markedly decreased and a pigment epithelial detachment resolved at 1 month after SRT treatment. Complete resolution of SRF was shown on OCT up to 6 months after SRT treatment. (C) 3, (D) 4, (E) 5, and (F) 6 months, respectively. There was no notable treatment-related retinal pigment epithelial damage.
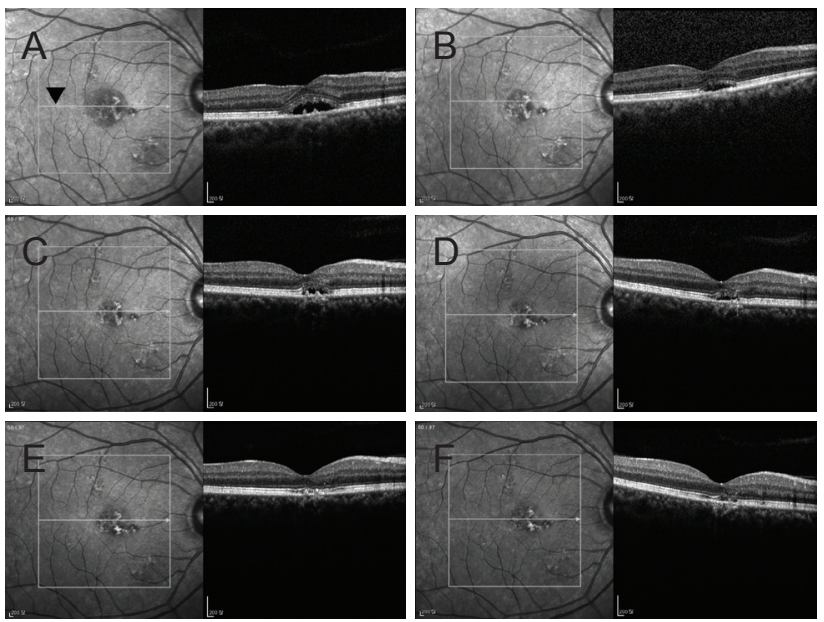

Fig. 3. Representative case of control arm (subject 08-07). A 50-year-old man with a 48-week history of subretinal fluid (SRF) in the right eye. Best-corrected visual acuity in the right eye was $20 / 25$ at baseline. SRF was observed at baseline by optical coherence tomography. (A) The height of SRF was $122.5 \mu \mathrm{m}$ at the baseline. Black arrowhead indicates the line where OCT scan was performed. (B) The height of SRF was $71.5 \mu \mathrm{m}$ after one month from baseline. (C) The SRF was not resolved naturally for 3 months, when the patient was treated by the selective retina therapy (SRT) laser system. (D) At month 4, 1 month following SRT treatment, the height of SRF decreased to $63 \mu \mathrm{m}$. (E,F) SRF was completely resolved two months following SRT treatment and it was maintained at the month 6 study exit visit, that is three months following treatment. There was no observable treatment-related retinal pigment epithelial damage. 
quently observed in SRT group (80.6\%) than in the sham group (44.1\%) at month 1 . SRF increased in 7 eyes of the sham group. However, only one eye showed increased SRF in SRT group at month 1. At month 3, degree of resolution of SRF was not significantly different between SRT and sham groups $(p=0.310)$. Representative cases from the SRT group and control group are presented in Fig. 2A-2F and $3 \mathrm{~A}-3 \mathrm{~F}$.

\section{Comparison of changes in OCT parameters and BCVA between SRT and sham groups}

At baseline, the peak height and volume of SRF, CMT, and TMV were not different between SRT and sham groups. At month 1, the peak height of SRF and CMT were significantly greater in the sham group than in SRT group ( $p=0.012, p=0.003$ ) (Fig. 4). At month 3, however, the difference was not significant. BCVA was not different between SRT and sham groups at baseline and at month 1 . At month 3, BCVA improved from $0.18 \log$ MAR to $0.10 \log$ MAR in SRT group $(p=0.001)$. And it was better than 0.12 $\operatorname{logMAR}$ in the sham group. However, the difference was in borderline significance $(p=0.054)$. This comparison is presented in Table 3.

Mixed model for repeated measures showed that changes in the peak height of SRF and CMT at the month 1 observation was significantly different between SRT group and the sham group (least squares mean difference, $-59.6 \mu \mathrm{m}$; 95\% confidence interval, -98.2 to $-21.2 ; p=0.0029$; least squares mean difference $-67.0 \mu \mathrm{m} ; 95 \%$ confidence interval, -104.8 to $-29.2 ; p=0.0007$, respectively) (Table 4). However, the differences were not significant at the month 3 .

\section{Removal of leakage and recurrence}

The removal rate of leakage between the SRT group and the sham group excluding control arm subjects who received SRT at month 3 was compared based on fluorescein angiography at 6 months (Table 5). The difference in percentage of subjects with complete removal of leakage was not significant between groups. The majority of subjects had no recurrence of SRF at 6 months after the SRT and sham procedures, $93.5 \%$ and $85.7 \%$, respectively; the difference was not significant. Recurrence rate was small at 6 months, $6.5 \%$ and $14.3 \%$, in the SRT group and sham group respectively.

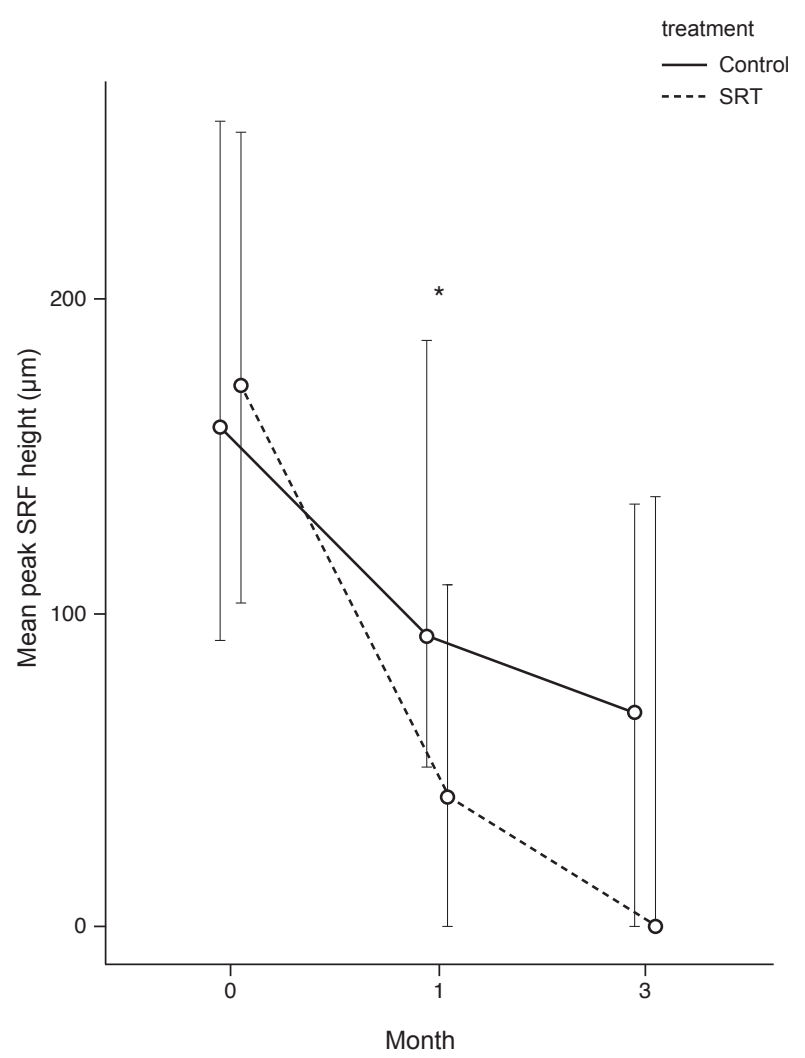

Fig. 4. Peak height of subretinal fluid (SRF) measured by optical coherence tomography. Peak SRF height was significantly higher in selective retina therapy (SRT) group than in control group at 1 month after treatment. Error bars indicate 95\% confidence interval. ${ }^{*} p<0.05$.

\section{Subgroup analysis for acute and chronic groups}

Median symptom duration was 7.0 months (range, 3.1 to 122.2 months). We classified patients into two subgroups based on their symptom duration; acute $(<6$ months of symptom duration) and chronic ( $\geq 6$ months of symptom duration). Among 68 patients, 31 had $<6$ months of symptom duration and the other 37 had $\geq 6$ months of symptom duration (Table 6). Among 31 patients in acute group, 19 underwent the SRT procedure while 12 underwent the sham procedure. Baseline peak height and volume of SRF, CMT, and TMV were not different between SRT and sham groups. At month 1, however, the peak height and volume of SRF, CMT, and TMV were smaller in SRT group than the sham group ( $p=0.006, p=0.011, p=0.014, p=0.033$, respectively). BCVA was not different between SRT and sham groups at baseline and at month 1 . However, at month 3, BCVA was better in SRT group than in the sham 
Table 3. Comparison of optical coherence tomography parameters and BCVA between the SRT group and the sham group

\begin{tabular}{|c|c|c|c|}
\hline & SRT group $(n=31)$ & Sham group $(n=37)$ & $p$-value ${ }^{*}$ \\
\hline \multicolumn{4}{|l|}{ At baseline } \\
\hline Peak height of SRF $(\mu \mathrm{m})$ & $172.0(103.0-253.0)$ & $159.0(91.3-256.3)$ & 0.664 \\
\hline SRF volume $\left(\mathrm{mm}^{3}\right)$ & $0.488(0.213-1.183)$ & $0.313(0.143-1.088)$ & 0.301 \\
\hline $\mathrm{CMT}(\mu \mathrm{m})$ & $393.0(321.0-477.0)$ & $396.0(298.5-496.5)$ & 0.907 \\
\hline $\mathrm{TMV}\left(\mathrm{mm}^{3}\right)$ & $9.90(9.10-11.10)$ & $9.50(8.95-10.40)$ & 0.267 \\
\hline BCVA (logMAR) & $0.18(0.10-0.34)$ & $0.18(0.10-0.40)$ & 0.580 \\
\hline \multicolumn{4}{|l|}{ At month 1} \\
\hline Peak height of SRF ( $\mu \mathrm{m})$ & $41.5(0-109.0)$ & $92.3(50.8-186.6)$ & $0.012^{\dagger}$ \\
\hline SRF volume $\left(\mathrm{mm}^{3}\right)$ & $0.027(0-0.357)$ & $0.175(0.043-0.583)$ & 0.096 \\
\hline $\mathrm{CMT}(\mu \mathrm{m})$ & $246.0(221.0-288.0)$ & $301.5(250.8-399.0)$ & $0.003^{\dagger}$ \\
\hline $\operatorname{TMV}\left(\mathrm{mm}^{3}\right)$ & $9.20(8.50-10.30)$ & $9.30(8.78-10.30)$ & 0.545 \\
\hline BCVA (logMAR) & $0.14(0-0.20)$ & $0.14(0.10-0.40)$ & 0.251 \\
\hline \multicolumn{4}{|l|}{ At month 3} \\
\hline Peak height of SRF $(\mu \mathrm{m})$ & $0(0-136.5)$ & $68.0(0-134.5)$ & 0.236 \\
\hline SRF volume $\left(\mathrm{mm}^{3}\right)$ & $0(0-0.448)$ & $0.072(0-0.273)$ & 0.425 \\
\hline $\mathrm{CMT}(\mu \mathrm{m})$ & $247.0(226.0-305.0)$ & $265.0(236.5-339.0)$ & 0.148 \\
\hline $\operatorname{TMV}\left(\mathrm{mm}^{3}\right)$ & $9.30(8.70-10.20)$ & $9.00(8.65-9.95)$ & 0.423 \\
\hline BCVA (logMAR) & $0.10(0-0.20)$ & $0.12(0.02-0.30)$ & 0.054 \\
\hline \multicolumn{4}{|l|}{ At month $6^{\ddagger}$} \\
\hline Peak height of SRF $(\mu \mathrm{m})$ & $0(0-49.0)$ & $0(0-0)$ & 0.419 \\
\hline SRF volume $\left(\mathrm{mm}^{3}\right)$ & $0(0-0.050)$ & $0(0-0)$ & 0.403 \\
\hline CMT $(\mu \mathrm{m})$ & $241.0(223.0-265.0)$ & $238.5(203.3-259.5)$ & 0.562 \\
\hline $\operatorname{TMV}\left(\mathrm{mm}^{3}\right)$ & $9.20(8.55-10.05)$ & $8.85(8.40-10.13)$ & 0.543 \\
\hline BCVA (logMAR) & $0(0-0.12)$ & $0.09(-0.10-0.13)$ & 0.966 \\
\hline
\end{tabular}

Values are presented as median (interquartile range).

$\mathrm{BCVA}=$ best-corrected visual acuity; SRT $=$ selective retina therapy; SRF = subretinal fluid; CMT = central macular thickness; TMV = total macular volume; logMAR $=$ logarithm of the minimum angle of resolution.

${ }^{*}$ Mann-Whitney $U$-test; ${ }^{\dagger} p<0.05$; ${ }^{*}$ Excluding missing or crossover subjects in the SRT group $(\mathrm{n}=29)$ and in the sham groups $(\mathrm{n}=12)$.

group ( $p=0.048)$. In 37 chronic symptomatic patients, there was no significantly different parameters between SRT and sham groups at both month 1 and 3.

\section{Contrast sensitivity}

Spatial frequency of 3.0, 6.0, 12.0, 18.0 cycles per degree, and mean sensitivity were analyzed. Change from baseline to 3 months and to 6 months were compared between SRT group and control. Crossover subjects were exculuded in this comparison. Contrast sensitivity was improved significantly in all the spatial frequency at month 3 and month 6 from baseline in both groups. However, the sensitivity im- provement did not show statistically significant difference between study groups (Supplemental Table 1).

\section{Adverse events}

Of the 68 participants randomized and treated in this study, 13 subjects reported a total 20 treatment emergent adverse events (TEAEs); none of the reported TEAEs were considered serious and none resulted in study discontinuation (Table 7). In the SRT group, 6 subjects reported a total 13 TEAEs while seven subjects reported a total 7 TEAEs in the sham group. Eye disorders were reported most frequently in either group $(n=4)$; followed by respiratory, 
Table 4. Comparison of changes in SRF and CMT

\begin{tabular}{|c|c|c|c|c|c|c|}
\hline & Comparison & $\begin{array}{l}\text { No. of } \\
\text { subjects }\end{array}$ & LSM & $\begin{array}{l}\text { LSM difference } \\
\text { (SE) }\end{array}$ & $\begin{array}{c}\text { LSM difference } \\
95 \% \mathrm{CI}\end{array}$ & $p$-value \\
\hline \multicolumn{7}{|l|}{ Peak height of SRF $(\mu \mathrm{m})$} \\
\hline 1 Month change from baseline & SRT vs. sham & 31 vs. 34 & -125.9 vs. -66.3 & $-59.7(19.3)$ & -98.2 to -21.2 & $0.0029^{\dagger}$ \\
\hline 3 Months change from baseline & SRT vs. sham & 31 vs. 37 & -122.5 vs. -102.3 & $-20.2(21.5)$ & -63.1 to 22.7 & 0.3509 \\
\hline \multicolumn{7}{|l|}{$\mathrm{CMT}(\mu \mathrm{m})$} \\
\hline 1 Month change from baseline & SRT vs. sham & 31 vs. 34 & -139.9 vs. -72.9 & $-67.0(18.9)$ & -104.8 to -29.2 & $0.0007^{\dagger}$ \\
\hline 3 Months change from baseline & SRT vs. sham & 31 vs. 37 & -130.6 vs. -110.6 & $-20.0(19.4)$ & -58.8 to 18.8 & 0.3061 \\
\hline
\end{tabular}

$\mathrm{SRF}=$ subretinal fluid; $\mathrm{CMT}=$ central macular thickness; $\mathrm{LSM}=$ least squares mean; $\mathrm{SE}=$ standard error; $\mathrm{CI}=$ confidence interval; $\mathrm{SRT}$ $=$ selective retina therapy.

"Mixed model for repeated measures. The LSM estimates are derived from an mixed model for repeated measures model with treatment, month, treatment-by-month interactions as factors, and baseline response as covariate using an unstructured covariance structure. The Kenward-Roger approximation is used to estimate denominator degrees of freedom; ${ }^{\dagger} p<0.05$.

Table 5. Comparison of removal of leakage and recurrence of subretinal fluid between the SRT group and the sham group excluding crossover patients

\begin{tabular}{|c|c|c|c|}
\hline & SRT group $(n=31)$ & Sham group $(n=14)$ & $p$-value \\
\hline \multicolumn{4}{|c|}{ Complete removal of leakage } \\
\hline Yes : no & $13: 18(41.9: 58.1)$ & $5: 8(35.7: 57.1)$ & 1.000 \\
\hline \multicolumn{4}{|l|}{ Recurrence } \\
\hline Yes : no & $2: 29(6.5: 93.5)$ & $2: 12(14.3: 85.7)$ & 0.578 \\
\hline
\end{tabular}

Values are presented as number (\%); Excluding missing data in the sham groups ( $\mathrm{n}=11$ for acute patients and $\mathrm{n}=23$ for chronic patients). $\mathrm{SRT}=$ selective retina therapy.

*Fisher exact test.

thoracic and mediastinal disorders $(\mathrm{n}=3)$; and vascular disorders $(n=3)$. Dry eye was the single most frequently reported TEAE among subjects in either group (two subjects in SRT group and one in sham group) followed by hypertension (two subjects in control). There was no case developed choroidal neovascularization or hemorrhage. All other TEAEs had single incidence. There were no serious adverse events reported in the study.

\section{Discussion}

This was a masked, randomized, controlled clinical trial to evaluate the efficacy of SRT using a Q-switched Nd: YLF laser (R:GEN) with RTF system iin patients with CSC. We found that more than half of the subjects treated with SRT achieved complete resolution of SRF at 3 months after the treatment while only a third of subjects in the untreated control group did. Even though the difference trended in favor of the SRT group, the difference was not statistically significant. Measuring SRF volume was performed on entire cube scans of spectral domain OCT and complete resolution was defined when the volume of SRF was estimated below $0.01 \mathrm{~mm}^{3}$. Volume based SRF measurement using multiple OCT scans has both strength and weakness currently. This method is so sensitive that even small amount of fluid is detected as remaining SRF. Volumetric analysis of SRF in AMD using artificial intelligence was able to detect fluid at nanoliter level, which was impossible by analysis of cross sectional OCT image [11]. This sensitivity of pixelwise-volume-based measurement might explain that the complete resolution of SRF was noted only less than half of patients while SRF decreased in more than $80 \%$ of patients. This low incidence of complete resolution could prevent the difference between SRT and control groups to reach the statistical significance because substantial, clinical meaningful reductions in fluid in CSC patients is not considered by this evaluation criterion. And 
Table 6. Comparison of parameters between the SRT group and the sham group in acute and chronic symptomatic patients

\begin{tabular}{|c|c|c|c|c|c|c|}
\hline & \multicolumn{3}{|c|}{ Acute $(n=31)$} & \multicolumn{3}{|c|}{ Chronic $(\mathrm{n}=37)$} \\
\hline & $\begin{array}{l}\text { SRT group } \\
(\mathrm{n}=19)\end{array}$ & $\begin{array}{l}\text { Sham group } \\
\quad(\mathrm{n}=12)\end{array}$ & $p$-value ${ }^{*}$ & $\begin{array}{l}\text { SRT group } \\
(\mathrm{n}=12)\end{array}$ & $\begin{array}{l}\text { Sham group } \\
\quad(\mathrm{n}=25)\end{array}$ & $p$-value \\
\hline \multicolumn{7}{|l|}{ At baseline } \\
\hline Peak height of SRF $(\mu \mathrm{m})$ & $179.0(108.0-311.5)$ & $166.0(85.4-288.9)$ & 0.459 & $140.3(76.6-225.1)$ & $143.0(99.8-252.5)$ & 0.532 \\
\hline SRF volume $\left(\mathrm{mm}^{3}\right)$ & $0.583(0.213-1.157)$ & $0.409(0.171-0.966)$ & 0.535 & $0.469(0.195-1.194)$ & $0.265(0.128-1.184)$ & 0.575 \\
\hline $\mathrm{CMT}(\mu \mathrm{m})$ & $407.0(335.0-513.0)$ & $406.0(289.5-474.3)$ & 0.435 & $341.5(290-430.0)$ & $386.0(319.0-505.0)$ & 0.344 \\
\hline $\mathrm{TMV}\left(\mathrm{mm}^{3}\right)$ & $10.20(9.20-11.50)$ & $10.3(9.20-11.73)$ & 0.795 & $9.45(8.90-10.88)$ & $9.30(8.70-9.90)$ & 0.491 \\
\hline BCVA (logMAR) & $0.10(0.08-0.26)$ & $0.18(0.25-0.38)$ & 0.675 & $0.25(0.10-0.50)$ & $0.20(0.10-0.40)$ & 0.643 \\
\hline \multicolumn{7}{|l|}{ At month $1^{\dagger}$} \\
\hline Peak height of SRF $(\mu \mathrm{m})$ & $41.5(0-85.8)$ & $153.0(62.0-184.5)$ & $0.006^{\ddagger}$ & $46.3(21.3-127.8)$ & $88.0(23.5-193.0)$ & 0.420 \\
\hline SRF volume $\left(\mathrm{mm}^{3}\right)$ & $0.018(0-0.316)$ & $0.423(0.047-0.650)$ & $0.011^{\ddagger}$ & $0.038(0.002-1.157)$ & $0.118(0.010-0.469)$ & 0.878 \\
\hline $\mathrm{CMT}(\mu \mathrm{m})$ & $245.0(225.0-270.0)$ & $351.0(259.0-408.0)$ & $0.014^{\ddagger}$ & $252.0(213.0-341.8)$ & $284.0(250.0-384.0)$ & 0.132 \\
\hline $\operatorname{TMV}\left(\mathrm{mm}^{3}\right)$ & $9.10(8.50-10.40)$ & $9.80(9.50-10.9)$ & $0.033^{\ddagger}$ & $9.35(8.50-10.30)$ & $8.90(8.60-9.70)$ & 0.595 \\
\hline BCVA (logMAR) & $0.10(0-0.20)$ & $0.10(0-0.30)$ & 0.704 & $0.20(0.10-0.29)$ & $0.20(0.10-0.42)$ & 0.936 \\
\hline \multicolumn{7}{|l|}{ At month 3} \\
\hline Peak height of SRF $(\mu \mathrm{m})$ & $0(0-70.0)$ & $44.8(0-122.6)$ & 0.412 & $71.0(0-154.9)$ & $86.5(0-137.5)$ & 0.886 \\
\hline SRF volume $\left(\mathrm{mm}^{3}\right)$ & $0(0-0.252)$ & $0.032(0-0.253)$ & 0.389 & $0.160(0-0.726)$ & $0.074(0-0.402)$ & 0.643 \\
\hline $\mathrm{CMT}(\mu \mathrm{m})$ & $240.0(226.0-276.0)$ & $249.5(222.8-332.5)$ & 0.675 & $262.5(212.5-348.3)$ & $285.0(242.5-344.5)$ & 0.413 \\
\hline $\operatorname{TMV}\left(\mathrm{mm}^{3}\right)$ & $9.10(8.70-10.20)$ & $9.05(8.83-10.6)$ & 0.765 & $9.55(8.48-10.20)$ & $9.00(8.50-9.85)$ & 0.344 \\
\hline BCVA (logMAR) & $0.06(0-0.10)$ & $0.15(0-0.30)$ & 0.048 & $0.15(0.01-0.28)$ & $0.12(0.07-0.35)$ & 0.962 \\
\hline
\end{tabular}

$\mathrm{SRT}=$ selective retina therapy; $\mathrm{SRF}=$ subretinal fluid; $\mathrm{CMT}=$ central macular thickness; $\mathrm{TMV}=$ total macular volume; $\mathrm{BCVA}=$ best-corrected visual acuity; $\log \mathrm{MAR}=$ logarithm of the minimum angle of resolution.

${ }^{*}$ Mann-Whitney $U$-test; ${ }^{\dagger}$ Excluding missing data in the sham groups $\left(\mathrm{n}=11\right.$ for acute patients and $\mathrm{n}=23$ for chronic patients); ${ }^{\star} p<0.05$.

on the other hand, current macular OCT only covers central area macula, which can underestimate the SRF beyond the OCT coverage.

Moreover, higher self-resolution rate in control group might also have contributed to the lower difference of complete resolution rate when comparing the two groups. Although participants had clinical symptoms over 3 months and median symptom duration was 7 months, $45.6 \%$ of patients had symptom duration less than 6 months. Most acute CSC could be resolved within 6 months after onset of symptoms. And more than $1 / 3$ patients in the control group had self-resolution of SRF in the current study. Therefore, further study with larger numbers of patients or more carefully designed criteria are required to confirm SRT efficacy on CSC simultaneously minimizing self-resolution rate.

In the secondary outcome measures, this study showed that change of SRF and macular edema were more rapid in
SRT group than in the sham group at the month 1 observation. And significant reduction of SRF was more frequently observed in SRT group. Moreover, peak height of SRF and CMT decreased at month 1 visit. However, it was not different at month 3. This result implies that SRT could enable faster absorption of SRF. Volume change from baseline shows better anatomical outcome at month 1 in the active treatment group. Although mechanism of SRT is poorly understood, it is reported that sublethal laser treatment increases the expression of heat shock proteins restores the RPE function [12]. This might accelerate the absorption of SRF and can be possible theoretic background of SRT efficacy without visible damage.

We further classified patients into acute and chronic subgroups. SRF parameters (peak height of SRF, SRF volume, CMT, and TMV) improved significantly in the acute subgroup at month 1. However, BCVA was better in acute active treatment subgroup than in the acute sham subgroup 
Table 7. Comparison of adverse events between SRT and sham groups

\begin{tabular}{|c|c|c|c|}
\hline & SRT group $(\mathrm{n}=31)$ & Sham group $(n=37)$ & Total $(n=68)$ \\
\hline \multicolumn{4}{|l|}{ Eye disorders } \\
\hline Blepharitis & $1(3.2)$ & 0 & $1(1.5)$ \\
\hline Conjunctival hemorrhage & $1(3.2)$ & 0 & $1(1.5)$ \\
\hline Conjunctivitis allergic & $1(3.2)$ & 0 & $1(1.5)$ \\
\hline Dry eye & $2(6.5)$ & $1(2.7)$ & $3(4.4)$ \\
\hline Vision blurred & 0 & $1(2.7)$ & $1(1.5)$ \\
\hline \multicolumn{4}{|l|}{ Systemic disorders } \\
\hline Abdominal pain & $1(3.2)$ & 0 & $1(1.5)$ \\
\hline Diarrhea & $1(3.2)$ & 0 & $1(1.5)$ \\
\hline Hepatitis & 0 & $1(2.7)$ & $1(1.5)$ \\
\hline Hypersensitivity & 0 & $1(2.7)$ & $1(1.5)$ \\
\hline Gastroenteritis & $1(3.2)$ & 0 & $1(1.5)$ \\
\hline Nasopharyngitis & $1(3.2)$ & 0 & $1(1.5)$ \\
\hline Asthma & $1(3.2)$ & 0 & $1(1.5)$ \\
\hline Cough & $1(3.2)$ & 0 & $1(1.5)$ \\
\hline Oropharyngeal pain & $1(3.2)$ & 0 & $1(1.5)$ \\
\hline Urticaria & $1(3.2)$ & 0 & $1(1.5)$ \\
\hline Cryoglobulinemia & 0 & $1(2.7)$ & $1(1.5)$ \\
\hline Hypertension & 0 & $2(5.4)$ & $2(2.9)$ \\
\hline
\end{tabular}

Values are presented as number (\%).

$\mathrm{SRT}=$ selective retina therapy.

at month 3. This result suggests that SRT could help in more rapid absorption of SRF in acute CSC. Generally current primary treatment option would be observation and waiting for spontaneous resolution for several months in acute cases. Early intervention has been sometimes recommended for early resolution of SRF in certain acute cases such as occupational need or prior poor prognosis of fellow eye. Adaptive optics scanning laser ophthalmoscopy showed abnormal cone mosaic pattern and reduced density even in retina of resolved CSC patients preserving more than 20 / 20 vision and ellipsoid zone structure [5]. And outer nuclear layer was decreased even in patients having symptom duration less than 1 month [13]. These results support active intervention is helpful even in acute CSC. SRT may be a good option for more rapid resolution of SRF and recovery of vision in patients with acute CSC.

Peak height of SRF used in current study has been suggested as useful representative value for estimated volume in previous studies [14]. Peak height was well matched to CMT. This can be explained as highest SRF was collected under fovea in most cases. We also analyzed SRF volume calculated from peak height at each scan. Although this is a sensitive method, SRF volume can be under-evaluated when SRF extends beyond OCT scan area $(6 \times 6 \mathrm{~mm})$. SRF change measured by above mentioned parameters was not related to visual acuity. This disagreement between visual acuity and SRF is also observed in age related macular degeneration and CSC [15]. Additionally, visual acuity measurement also has limitation of reflecting whole visual function. Metamorphopsia test or microperimetry can be complementary visual function examination methods to evaluate other than central vision. Further study using these examinations can reveal the subtle improvement or deterioration of visual function after treatment of CSC patients using SRT.

Several studies have shown beneficial effects of SRT in CSC. Complete resolution of SRF was observed in about $75 \%$ of chronic CSC $[9,10]$. Kim et al. [16] reported that SRF was completely resolved after SRT after 3 months in acute CSC. Different type of micropulse laser system, sub- 
threshold micropulse laser therapy, have also been studied as CSC treatment [17-19]. These data also suggested that subthreshold laser is effective in acute and chronic CSC. Wavelength is different and feedback system is absent in these subthreshold laser systems. Histopathological study has not revealed any visible difference at subthreshold laser irradiated lesion. However, SRT irradiated area is visible on fluorescein angiogram. It is feasible that efficacy can be different between SRT and subthreshold laser systems. There were no clinical trials where a comparative analysis between these different laser systems had been performed until recently.

The PLACE trial is the largest prospective randomized controlled study using subthreshold micropulse laser treatment in CSC [20]. This was comparative study to evaluate the subthreshold micropulse laser and PDT. PDT showed better functional and anatomical outcome in chronic CSC at 6 to 8 weeks and 7 to 8 months after first treatment. Anatomical outcome was complete resolution of SRF and visual function include vision and retinal sensitivity in microperimetry. The authors presumed that choroidal hyperpermeability may play a greater role in CSC pathophysiology and this may explain the better outcome using PDT. Multiple studies reported the efficacy of PDT in resolving CSC [21]. However, RPE dysfunction is also suggested as etiology of CSC. Because SRT targets RPE lesion directly, there may be certain patients group who could show better outcome after SRT. In our study, the subgroup with a shorter symptom duration showed better anatomical and functional outcome. Further study to find optimal candidates for SRT in CSC is required. In spite of favorable safety profile of reduced PDT, RPE atrophy and acute severe visual decline were observed in $4 \%$ and $1.5 \%$, respectively [21]. Besides these rare severe adverse events, required protection from sunlight exposure is weakness of PDT in treating CSC, considering higher incidence rate of CSC in middle aged working population.

As alternative treatment option other than PDT for CSC, direct laser photocoagulation, mineralocorticoid receptor antagonists and anti VEGF agents have been used. Indication of direct laser is limited to extrafoveal area because of neuroretinal damage. Efficacy of mineralocorticoid receptor antagonists in CSC is questionable [22,23]. Moreover, close monitoring for hormonal and blood potassium monitoring is required to prevent severe systemic complication. Several studies showed efficacy of anti VEGF agents in
CSC. However, overall treatment usefulness is questionable. Systematic meta-analysis failed to show efficacy of anti VEGF agents in CSC [24]. Moreover, intravitreal injections have a small but serious risk of endophthalmitis.

In this trial we used SRT with RTF system. This system has not been reported to decrease retinal sensitivity even in cases undergoing retreatment, when retinal sensitivity is evaluated using microperimetry [9]. In the current study, reduction of contrast sensitivity was not observed in treatment group and change was not different from control. Participants did not experience any laser related adverse event. SRT using a Q-switched Nd: YLF laser (R:GEN) with RTF system suggested that it would be safe and effective for early resolution of SRF in patients with acute CSC. However, in this study we included patients with clinical symptoms over 3 months, therefore further study targeting patients with shorter symptom duration would be necessary to confirm the effect of SRT with feedback system on acute CSC.

This study has several limitations. The first one is the small number of subjects. In part this limitation is that the study sample size did not afford adequate power for any subgroup analyses. The second limitation comes from the definition of complete resolution of SRF which was determined by using OCT scan covering limited macular area. We defined complete resolution of SRF when the volume of SRF by OCT was estimated as zero. This not only did not allow for considering substantial decreases in fluid in this central area as being meaningful from a primary analysis perspective, it was not also possible to detect fluid outside the OCT scan area for analyses. To account for substantial decreases in fluid we have included reduction in the peak height of the SRF that is located within the measurement area. And finally, the short follow-up period was also another limitation of this study. Although two different model of OCT devices were used in this study, outcome measures is not likely to be biased by different measuring algorithm of each devices. Only one site used the 3D-OCT 1000 device and 20 patients were analyzed by this device. SRT and control group was $10: 10$ in this site. Our outcome measures were decrease of SRF and change of OCT parameters. Same device was used in each patient for the whole study duratione. Therefore, effect of different OCT devices are expected to be limited.

In conclusion, this sham controlled randomized clinical trial showed SRT with feedback system showed more 
rapidresolution of SRF at 1 month in patients with CSC. And structural improvement was prominent in acute cases based on subgroup analyses. Moreover, there was no treatment associated adverse event in either the SRT or control treatments including in those subjects who were in the control group and were eligible for and received SRT treatment at month 3. This study provides evidence that SRT with feedback system under the study conditions may be considered as a safe and effective treatment option for acute CSC especially in cases with center involved fluid.

\section{Conflict of Interest}

Though this study was supported by Lutronic Vision, Inc., the collection, the analysis, and the interpretation of data had been done independently by authors.

\section{Acknowledgements}

This study was supported by Lutronic Vision, Inc. and a grant from the Korea Health Technology R\&D Project through the Korea Health Industry Development Institute (KHIDI), funded by the Ministry of Health \& Welfare, Republic of Korea (grant no. HI15C0892).

This work was partially supported by the Korean Association of Retinal Degeneration.

\section{Supplemental Material}

Supplemental Table 1 is available from: https://doi. org/10.3341/kjo.2020.0112.

\section{References}

1. Rim TH, Kim HS, Kwak J, et al. Association of corticosteroid use with incidence of central serous chorioretinopathy in South Korea. JAMA Ophthalmol 2018;136:1164-9.

2. Daruich A, Matet A, Dirani A, et al. Central serous chorioretinopathy: recent findings and new physiopathology hypothesis. Prog Retin Eye Res 2015;48:82-118.

3. Fok AC, Chan PP, Lam DS, Lai TY. Risk factors for recurrence of serous macular detachment in untreated patients with central serous chorioretinopathy. Ophthalmic Res 2011;46:160-3.

4. Matet A, Daruich A, Zola M, Behar-Cohen F. Risk factors for recurrences of central serous chorioretinopathy. Retina 2018;38:1403-14.

5. Ooto S, Hangai M, Sakamoto A, et al. High-resolution imaging of resolved central serous chorioretinopathy using adaptive optics scanning laser ophthalmoscopy. Ophthalmology 2010;117:1800-9.

6. Robertson DM. Argon laser photocoagulation treatment in central serous chorioretinopathy. Ophthalmology 1986;93:9724.

7. Roider J, Brinkmann R, Wirbelauer C, et al. Retinal sparing by selective retinal pigment epithelial photocoagulation. Arch Ophthalmol 1999;117:1028-34.

8. Elsner H, Porksen E, Klatt C, et al. Selective retina therapy in patients with central serous chorioretinopathy. Graefes Arch Clin Exp Ophthalmol 2006;244:1638-45.

9. Kang S, Park YG, Kim JR, et al. Selective retina therapy in patients with chronic central serous chorioretinopathy: a pilot study. Medicine (Baltimore) 2016;95:e2524.

10. Park YG, Kang S, Kim M, et al. Selective retina therapy with automatic real-time feedback-controlled dosimetry for chronic central serous chorioretinopathy in Korean patients. Graefes Arch Clin Exp Ophthalmol 2017;255:137583.

11. Schmidt-Erfurth U, Vogl WD, Jampol LM, Bogunovic H. Application of automated quantification of fluid volumes to anti-VEGF therapy of neovascular age-related macular degeneration. Ophthalmology 2020;127:1211-9.

12. Sramek C, Mackanos M, Spitler R, et al. Non-damaging retinal phototherapy: dynamic range of heat shock protein expression. Invest Ophthalmol Vis Sci 2011;52:1780-7.

13. Hata M, Oishi A, Shimozono M, et al. Early changes in foveal thickness in eyes with central serous chorioretinopathy. Retina 2013;33:296-301.

14. Ahn SE, Oh J, Oh JH, et al. Three-dimensional configuration of subretinal fluid in central serous chorioretinopathy. Invest Ophthalmol Vis Sci 2013;54:5944-52.

15. Chakravarthy U, Pillai N, Syntosi A, et al. Association between visual acuity, lesion activity markers and retreatment decisions in neovascular age-related macular degeneration. Eye (Lond) 2020;34:2249-56.

16. Kim YJ, Lee YG, Lee DW, Kim JH. Selective retina therapy with real-time feedback-controlled dosimetry for treating acute idiopathic central serous chorioretinopathy in 
Korean patients. J Ophthalmol 2018;2018:6027871.

17. Abd Elhamid AH. Subthreshold micropulse yellow laser treatment for nonresolving central serous chorioretinopathy. Clin Ophthalmol 2015;9:2277-83.

18. Roisman L, Magalhaes FP, Lavinsky D, et al. Micropulse diode laser treatment for chronic central serous chorioretinopathy: a randomized pilot trial. Ophthalmic Surg Lasers Imaging Retina 2013;44:465-70.

19. Arora S, Sridharan P, Arora T, et al. Subthreshold diode micropulse laser versus observation in acute central serous chorioretinopathy. Clin Exp Optom 2019;102:79-85.

20. van Dijk E, Fauser S, Breukink MB, et al. Half-dose photodynamic therapy versus high-density subthreshold micropulse laser treatment in patients with chronic central serous chorioretinopathy: the PLACE trial. Ophthalmology 2018;125:1547-55.
21. Lim JI, Glassman AR, Aiello LP, et al. Collaborative retrospective macula society study of photodynamic therapy for chronic central serous chorioretinopathy. Ophthalmology 2014;121:1073-8.

22. Wang SK, Sun P, Tandias RM, et al. Mineralocorticoid receptor antagonists in central serous chorioretinopathy: a meta-analysis of randomized controlled trials. Ophthalmol Retina 2019;3:154-60.

23. Lotery A, Sivaprasad S, O'Connell A, et al. Eplerenone for chronic central serous chorioretinopathy in patients with active, previously untreated disease for more than 4 months (VICI): a randomised, double-blind, placebo-controlled trial. Lancet 2020;395:294-303.

24. Ji S, Wei Y, Chen J, Tang S. Clinical efficacy of anti-VEGF medications for central serous chorioretinopathy: a meta-analysis. Int J Clin Pharm 2017;39:514-21. 
Supplemental Table 1. Contrast sensitivity change

\begin{tabular}{|c|c|c|c|c|c|}
\hline & SRT $(n=31)$ & $p$-value ${ }^{*}$ & Control $(n=14)$ & $p$-value ${ }^{\dagger}$ & $p$-value \\
\hline Baseline-spatial frequency $3.0 \mathrm{cpd}$ & $1.32 \pm 0.45$ & & $1.23 \pm 0.31$ & & 0.48 \\
\hline Change from baseline to 3 months & $0.20 \pm 0.34$ & 0.005 & $0.34 \pm 0.32$ & 0.001 & 0.19 \\
\hline Change from baseline to 6 months & $0.23 \pm 0.36$ & 0.002 & $0.39 \pm 0.35$ & $<0.001$ & 0.16 \\
\hline Baseline-spatial frequency $6.0 \mathrm{cpd}$ & $1.35 \pm 0.41$ & & $1.28 \pm 0.41$ & & 0.56 \\
\hline Change from baseline to 3 months & $0.23 \pm 0.32$ & $<0.001$ & $0.33 \pm 0.31$ & $<0.001$ & 0.31 \\
\hline Change from baseline to 6 months & $0.38 \pm 0.38$ & $<0.001$ & $0.37 \pm 0.34$ & $<0.001$ & 0.98 \\
\hline Baseline-spatial frequency $12.0 \mathrm{cpd}$ & $0.85 \pm 0.35$ & & $0.84 \pm 0.39$ & & 0.96 \\
\hline Change from baseline to 3 months & $0.29 \pm 0.39$ & $<0.001$ & $0.23 \pm 0.31$ & 0.013 & 0.56 \\
\hline Change from baseline to 6 months & $0.50 \pm 0.47$ & $<0.001$ & $0.43 \pm 0.34$ & $<0.001$ & 0.58 \\
\hline Baseline-spatial frequency $18.0 \mathrm{cpd}$ & $0.41 \pm 0.34$ & & $0.41 \pm 0.41$ & & 0.99 \\
\hline Change from baseline to 3 months & $0.28 \pm 0.43$ & 0.001 & $0.21 \pm 0.42$ & 0.075 & 0.58 \\
\hline Change from baseline to 6 months & $0.47 \pm 0.48$ & $<0.001$ & $0.34 \pm 0.44$ & 0.01 & 0.38 \\
\hline Baseline-spatial frequency average value & $0.98 \pm 0.33$ & & $0.94 \pm 0.34$ & & 0.7 \\
\hline Change from baseline to 3 months & $0.25 \pm 0.29$ & $<0.001$ & $0.28 \pm 0.23$ & $<0.001$ & 0.74 \\
\hline Change from baseline to 6 months & $0.39 \pm 0.34$ & $<0.001$ & $0.38 \pm 0.29$ & $<0.001$ & 0.92 \\
\hline
\end{tabular}

Values are presented as mean \pm standard deviation.

$\mathrm{SRT}=$ selective retina therapy; $\mathrm{cpd}=$ cycle per degree.

${ }^{*} t$-test compared to baseline value; ${ }^{\dagger}$ Mann-Whitney $U$-test compared to baseline value; ${ }^{\dagger}$ Mann-Whitney $U$-test between SRT group and non-crossover control group. 